\title{
A Direct Search Method to solve Economic Dispatch Problem with Valve-Point Effect
}

\author{
M.Narayanan ${ }^{1}$, S. Siva ${ }^{2}$, D. Velmurugan ${ }^{3}$ \\ ${ }^{1}$ M. Narayanan is currently working as Assistant Professor in Electrical \& Electronics Engg. Department, Info \\ Institute of Engineering, Coimbatore. \\ ${ }^{2}$ S. Siva is currently working as Assistant Professor in Electrical \& Electronics Engg. Department, Info Institute \\ of Engineering, Coimbatore. \\ ${ }^{3}$ D. Velmurugan is currently working as Assistant Professor in Electrical \& Electronics Engg. Department, Info \\ Institute of Engineering, Coimbatore.
}

\begin{abstract}
Scarcity of energy resources, increasing power generation cost and ever-growing demand for electric energy necessitates optimal economic dispatch in today's power systems. The main objective of economic dispatch is to reduce the total power generation cost, while satisfying various equality and inequality constraints. Traditionally in economic dispatch problems, the cost function for generating units has been approximated as a quadratic function which doesn't provide accurate results. Moreover, to obtain accurate fuel cost, valve-point effect in thermal power plant has to be taken into account. The inclusion of valve-point effect makes the modeling of the fuel cost functions of generating units more practical. In this paper a new evolutionary algorithm called Pattern Search Technique, has been employed to solve economic dispatch problem with the valve-point effect. Using this technique the non-linear cost function is solved for three unit system and the results are compared with the traditional PSO and GA method. These results prove that Pattern Search method is capable of getting higher quality solution including mathematical simplicity, fast convergence, and robustness to solve hard optimization problems.
\end{abstract}

Keywords: Economic Dispatch, Valve point effect, Pattern Search Method.

\section{Introduction}

Economic dispatch is one of the most important problems to be solved in the operation and planning of a power system. The primary objective of the electric power generation is to schedule the committed generating unit outputs so as to meet the required load demand at minimum operating cost while satisfying all unit and system equality and inequality constraints. In the traditional ED problem, the cost function for each generator has been approximately represented by a single quadratic function and is solved using mathematical programming based on the optimization techniques such as lambda-iteration method, gradient-based method, etc. These mathematical methods require incremental or marginal fuel cost curves which should be monotonically increasing to find global optimal solution.

The fuel cost functions of generating units can be modeled in a more practical fashion by including the valve-point effects. The valve-point effects result in the ripples in the fuel cost function; thereby the number of local optima is increased. Thus, the practical ED problem is represented as a non smooth optimization problem with equality and inequality constraints, which cannot be solved by the traditional mathematical methods. Dynamic programming method can solve such types of problems, but it suffers from so-called the curse of dimensionality. Over the past few years, in order to solve this problem, many salient methods have been developed such as genetic algorithm, evolutionary programming, tabu search, neural network approaches, and particle swarm optimization.

Considering stochastic search algorithms such as Genetic Algorithm GA[3], Simulated Annealing (SA),etc., Direct search methods[5], may prove to be very effective in solving nonlinear EDP problems without any restrictions on the shape of the cost curves. The first one don't guarantee in discovering the globally optimal solution in finite time, they often provide a fast and reasonable solution (sub-optimal near globally optimal). The SA method is a powerful optimization technique and it has the ability to find near global optimum solutions for the optimization problem. SA is applied in many power system problems. However, appropriate setting of the parameters of the SA based algorithm is a difficult task and the speed of the algorithm is slow when applied to a real power system.

Direct search (DS) methods are evolutionary algorithms used to solve constrained optimization problems. DS method do not require any information about the gradient of the objective function at hand, while searching for an optimum solution. This family includes Pattern Search (PS) algorithms, simplex methods (SM) (different from the simplex used in linear programming), Powell Optimization (PO) and others. The Pattern Search method is a technique that is suitable to solve a variety of optimization problems that lie outside the 
scope of the standard optimization methods. Generally, PS has the advantage of being very simple in concept, easy to implement and computationally efficient. Hence Pattern Search algorithm is used to solve power system economic load dispatch problem (ELD) with valve-point effect. The convergence characteristics and robustness of this method proves efficient when compared to other methods.

\subsection{Basic objective function}

\section{Problem Formulation}

The objective of the economic dispatch problem is to minimize the total fuel cost of thermal power plants subjected to the operating constraints of a power system. In general, it can be formulated mathematically with an objective function and two constraints as follows:

$$
\begin{gathered}
\mathrm{N} \\
\mathrm{F}_{\mathrm{T}}=\sum \mathrm{F}_{\mathrm{i}}\left(\mathrm{P}_{\mathrm{i}}\right) \\
\mathrm{i}=1 \\
\mathrm{~F}_{\mathrm{i}}\left(\mathrm{P}_{\mathrm{i}}\right)=\mathrm{a}_{\mathrm{i}} \mathrm{P}_{\mathrm{i}}^{2}+\mathrm{b}_{\mathrm{i}} \mathrm{P}_{\mathrm{i}}+\mathrm{c}_{\mathrm{i}}
\end{gathered}
$$

where,

$\mathrm{F}_{\mathrm{T}} \quad$ total generation cost,

$\mathrm{F}_{\mathrm{i}} \quad$ cost function of generator $\mathrm{i}$,

$a_{i}, b_{i}, c_{i}$ cost coefficients of generator $i$,

$\mathrm{P}_{\mathrm{i}} \quad$ power of generator $\mathrm{i}$,

$\mathrm{N} \quad$ number of generating units.

\subsection{The Constraints}

1) Equality or Active power balance equation

For power balance, an equality constraint should be satisfied. The total power generated should be equal to the sum of total load demand $\left(\mathrm{P}_{\mathrm{D}}\right)$ and the total transmission line loss $\left(\mathrm{P}_{\text {Loss }}\right)$.

$$
\underset{i=1}{\sum \mathrm{P}_{\mathrm{i}}=\mathrm{P}_{\mathrm{D}}+\mathrm{P}_{\text {Loss }}}
$$

However, the transmission loss is not considered in this paper for simplicity (i.e., $\mathrm{P}_{\text {Loss }}=0$ ).

\section{2) Minimum and maximum generation constraints}

Generation output of each generator should lie between maximum and minimum limits. The corresponding inequality constraints for each generator are

$$
\mathrm{P}_{\mathrm{i}, \min } \leq \mathrm{P}_{\mathrm{i}} \leq \mathrm{P}_{\mathrm{i}, \max }
$$

where $\mathrm{P}_{\mathrm{i}, \min }$ and $\mathrm{P}_{\mathrm{i}, \max }$ are the minimum and maximum output of generator $\mathrm{i}$, respectively.

\subsection{Valve-point effects}

The generating units with multi-valve steam turbines exhibit a greater variation in the fuel-cost functions. Since the valve point results in the ripples as shown in Fig. 1, a cost function contains higher order nonlinearity [9]. Therefore, the equation (2) should be replaced as the equation (5) to consider the valve-point effects. Here, the sinusoidal functions are thus added to the quadratic cost functions as follows.

$$
F_{i}\left(P_{i}\right)=a_{i} P_{i}^{2}+b_{i} P_{i}+c_{i}+\left|e_{i} \times \sin \left(f_{i} \times\left(P_{i, \min }-P_{i}\right)\right)\right|
$$

Where $e_{i}$ and $f_{i}$ are the coefficients of generator i reflecting valve-point effects. 


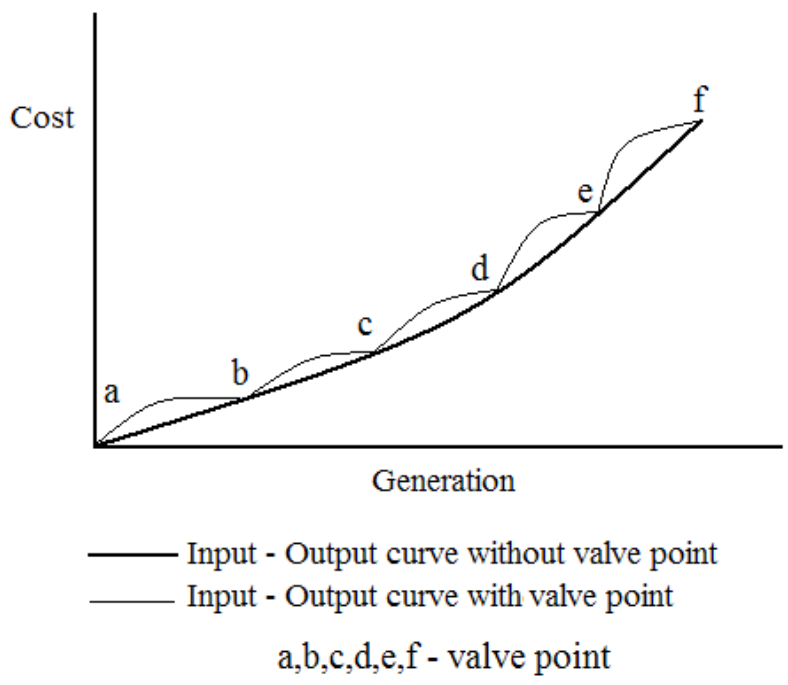

Fig. 1 Cost Curve for large steam power plant with valve-point effect

\section{Pattern Search Method}

The PS optimization routine is a derivative free evolutionary technique that is suitable to solve a variety of optimization problems that lie outside the scope of the standard optimization methods. Generally, PS has the advantage of being very simple in concept, and easy to implement and computationally efficient algorithm [11]. Unlike other heuristic algorithms, such as GA, PS possesses a flexible and well-balanced operator to enhance and adapt the global and fine tune local search.

The PS algorithm proceeds by computing a sequence of points that may or may not approach the optimal point. The algorithm starts by establishing a set of points called mesh, around the given point. This current point could be the initial starting point supplied by the user or it could be computed from the previous step of the algorithm. The mesh is formed by adding the current point to a scalar multiple of a set of vectors called a pattern. If a point in the mesh is found to improve the objective function at the current point, the new point becomes the current point at the next iteration.

This may be better explained by the following:

First Step: The Pattern search begins at the initial point $X_{0}$ that is given as a starting point by the user. At the first iteration, with a scalar of magnitude 1 called mesh size, the pattern vectors are constructed as [0 1], [1 0$]$, $\left[\begin{array}{ll}-1 & 0]\end{array}\right]$ and $[0-1]$, they may be called direction vectors. Then the PS algorithm adds the direction vectors to the initial point $\mathrm{X}_{0}$ to compute the following mesh points:

$\mathrm{X}_{0}+\left[\begin{array}{ll}1 & 0\end{array}\right]$

$\mathrm{X}_{0}+\left[\begin{array}{ll}0 & 1\end{array}\right]$

$\mathrm{X}_{0}+\left[\begin{array}{ll}-1 & 0]\end{array}\right.$

$\mathrm{X}_{0}+[0-1]$

Fig. 2. illustrates the formation of the mesh and pattern vectors. The algorithm computes the objective function at the mesh points in the order shown.

The algorithm polls the mesh points by computing their objective function values until it finds one whose value is smaller than the objective function value of $\mathrm{X}_{0}$. If there is such point, then the poll is successful and the algorithm sets this point equal to $\mathrm{X}_{1}$.

After a successful poll, the algorithm steps to iteration 2 and multiplies the current mesh size by 2, (this is called the expansion factor and has a default value of 2). The mesh at iteration 2 contains the following points: $X_{1}+2 *[10], X_{1}+2 *\left[\begin{array}{ll}0 & 1\end{array}\right], X_{1}+2 *[-10]$ and $X_{1}+2 *[0-1]$. The algorithm polls the mesh points until it finds one whose value is smaller than the objective function value of $X_{1}$. The first such point it finds is called $X_{2}$, and the poll is successful. As the poll is successful, the algorithm multiplies the current mesh size by 2 to get a mesh size of 4 at the third iteration. 


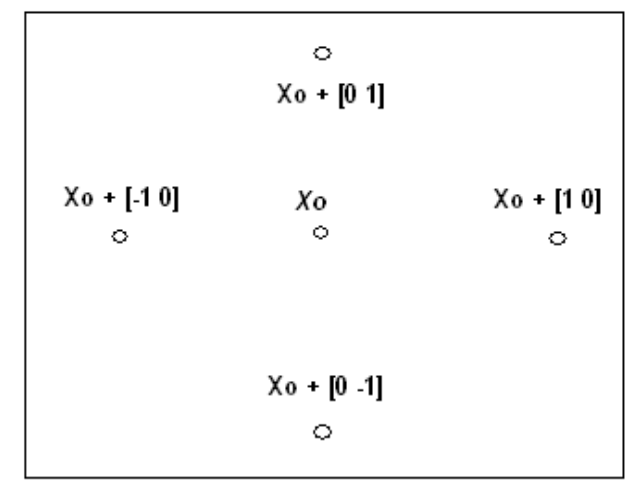

Fig. 2. PS Mesh points and Pattern

Second Step: Now if in iteration 3, none of the mesh points has a smaller objective function value than the value at $\mathrm{X}_{2}$, the poll is called an unsuccessful poll. In this case, the algorithm does not change the current point at the next iteration, i.e., $X_{3}=X_{2}$. At the next iteration, the algorithm multiplies the current mesh size by 0.5 , a contraction factor, so that the mesh size at the next iteration is smaller. The algorithm then polls with a smaller mesh size. The PS optimization algorithm will repeat the illustrated steps until it finds the optimal solution for the minimization of the objective function. The PS algorithm stops when any of the following conditions occurs:

- The mesh size is less than the mesh tolerance.

- The number of iterations performed by the algorithm reaches the value of maximum iteration number.

- The total number of objective function evaluations performed by the algorithm reaches the value of maximum function evaluations.

- The distance between the point found at one successful poll and the point found at the next successful poll is less than the specified tolerance.

- The change in the objective function from one successful poll to the next successful poll is less than the objective function tolerance.

All the parameters involved in the PS optimization algorithm can be pre-defined subject to the nature of the problem being solved.

\subsubsection{Flowchart of Pattern Search Method}

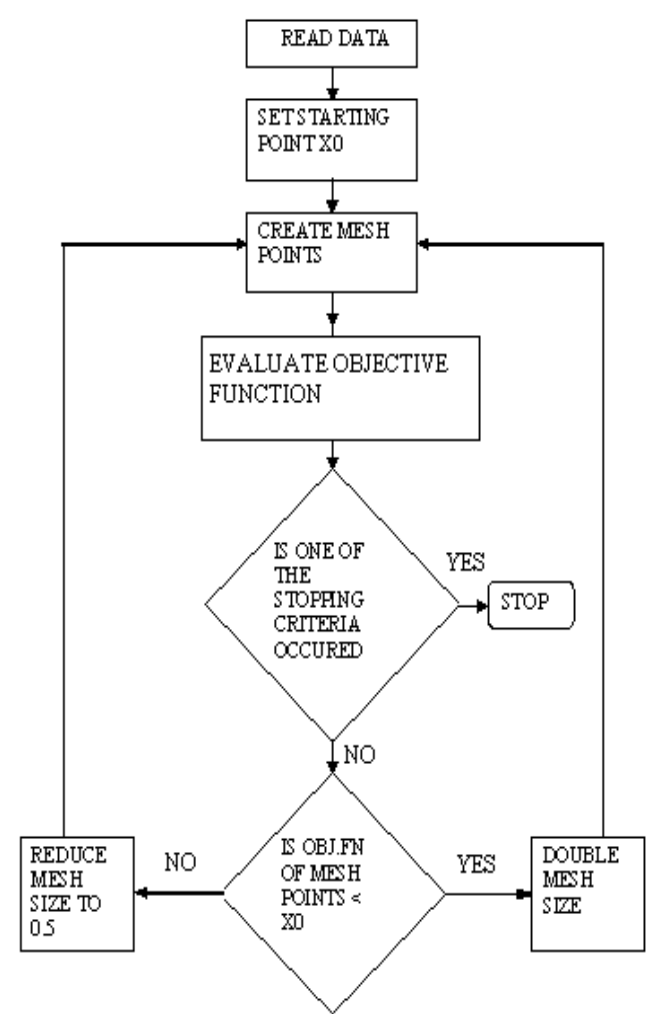

Fig. 3. PS Flowchart 
Step 1: Read the given data.

\section{Implementation of PS Algorithm}

Step 2: Set the starting or initial point X0.

Step 3: Create mesh points using direction vectors.

Step 4: Calculate the value of objective function for each mesh point.

Step 5: Check whether a stopping criterion occurred or not. If occurred goto step 8.

Step 6: Check whether the value of objective function using mesh point is than the starting point. If yes, double mesh size and goto step 3 .

Step 7: If no, reduce mesh size to 0.5 and go to step 3 .

Step 8: Stop.

\section{Simulated Result Analysis}

To verify the feasibility of the proposed PS method, three unit power systems were tested. The results obtained from the Pattern Search Method are compared with Particle Swarm Optimization (PSO) and Genetic Algorithm (GA).

This system comprises of 3 generating units and the input data of 3-generator system are given in Table I. Here, the total demand for the system is set to $850 \mathrm{MW}$.

Table I: Data For Test Case (3-Unit System)

\begin{tabular}{|l|l|l|l|l|l|l|l|}
\hline Unit & $\mathrm{a}_{\mathrm{i}}$ & $\mathrm{b}_{\mathrm{i}}$ & $\mathrm{c}_{\mathrm{i}}$ & $\mathrm{e}_{\mathrm{i}}$ & $\mathbf{f}_{\mathrm{i}}$ & $\mathbf{P}_{\mathrm{i}, \min }$ & $\mathrm{P}_{\mathrm{i}, \max }$ \\
\hline $\mathbf{1}$ & $\mathbf{5 6 1}$ & $\mathbf{7 . 9 2}$ & $\mathbf{0 . 0 0 1 5 6 2}$ & $\mathbf{3 0 0}$ & $\mathbf{0 . 0 3 1 5}$ & $\mathbf{1 0 0}$ & $\mathbf{6 0 0}$ \\
\hline $\mathbf{2}$ & $\mathbf{3 1 0}$ & $\mathbf{7 . 8 5}$ & $\mathbf{0 . 0 0 1 9 4 0}$ & $\mathbf{2 0 0}$ & $\mathbf{0 . 0 4 2 0}$ & $\mathbf{1 0 0}$ & $\mathbf{4 0 0}$ \\
\hline $\mathbf{3}$ & $\mathbf{7 8}$ & $\mathbf{7 . 9 7}$ & $\mathbf{0 . 0 0 4 8 2 0}$ & $\mathbf{1 5 0}$ & $\mathbf{0 . 0 6 3 0}$ & $\mathbf{5 0}$ & $\mathbf{2 0 0}$ \\
\hline
\end{tabular}

The obtained results for the 3-generator system using the Pattern Search Method are given in Table II and the results are compared with PSO and GA.

Table II: Comparison Of Simulation Results Of Ga, Pso And Ps Method Considering Valve-Point Effect (3-

\begin{tabular}{|l|l|l|}
\multicolumn{3}{|c}{ Unit System $)$} \\
\hline PARAMETERS & GA & PS \\
\hline Pg $_{1}(\mathrm{MW})$ & $\mathbf{3 9 6 . 6 1}$ & $\mathbf{4 9 8 . 9 3 2 4}$ \\
\hline $\mathbf{P g}_{2}(\mathrm{MW})$ & $\mathbf{2 5 3 . 8 1}$ & $\mathbf{2 5 1 . 2}$ \\
\hline $\mathbf{P g}_{3}(\mathrm{MW})$ & $\mathbf{1 9 9 . 5 9}$ & $\mathbf{9 9 . 8 6 6 5 5}$ \\
\hline Total fuel cost $(\$ / \mathrm{Hr})$ & $\mathbf{8 2 9 5 . 3 2 4 3}$ & $\mathbf{8 2 4 0 . 6 5 9 3}$ \\
\hline
\end{tabular}

\section{Pattern Search Plots}

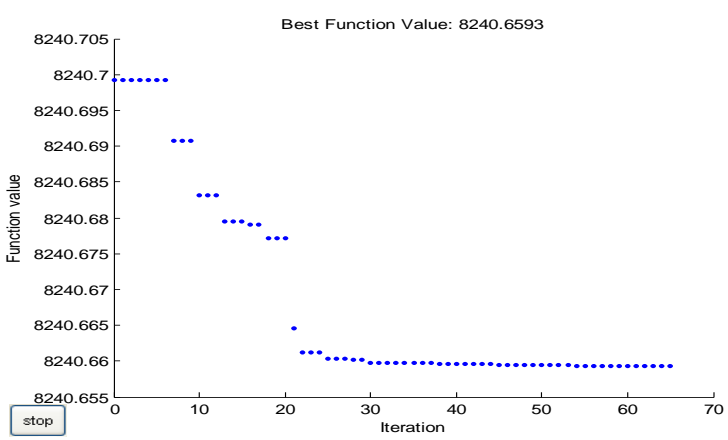

Fig. 4. Function value Vs Iteration 


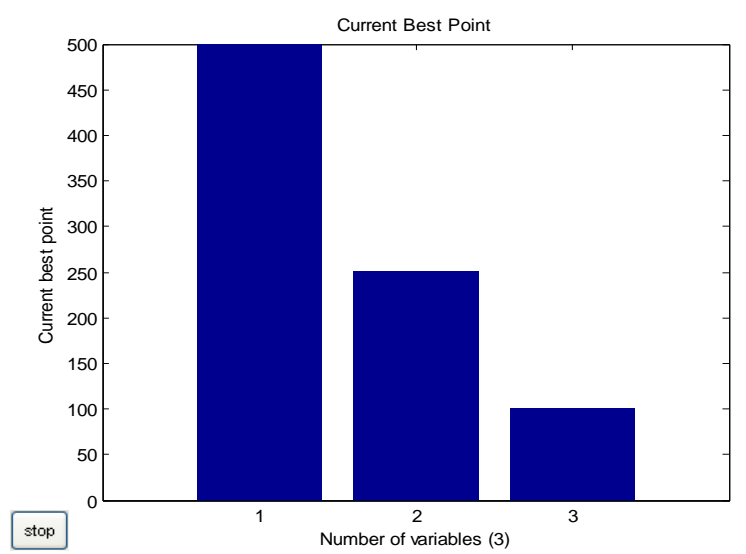

Fig. 5. Current best point Vs number of variables

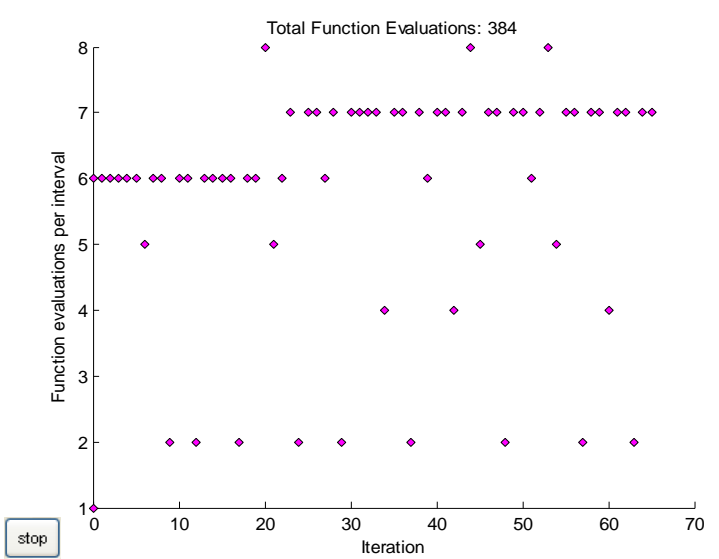

Fig. 6. Function evaluation per interval Vs Iteration

The plots obtained using Pattern Search technique by solving the cost function taking valve-point effect into account is shown in the Fig. 4, 5 and 6.

\section{Conclusion}

Pattern Search method for solving the economic dispatch problem with valve-point effect was presented. As the method is sensitive to starting point, it cannot find the required optimal solution in the first run. Therefore, to obtain optimal solution the program is made to run assuming the starting points randomly. Whenever there is an improvement in the solution in the PS run that will be taken as the starting point. The Pattern Search method is capable of dealing directly with load demand at various intervals of time in the scheduled horizon with no restrictions on the shape of the input-output cost function of the generating unit. It is very fast compared to other evolutionary techniques in exploring the search space. The result shows that the convergence property was not affected based on the shape of the incremental fuel cost function. The advantage of this method is its ability in finding high quality solutions reliably with fast converging characteristics.

The transmission loss is not considered in this work for simplicity. To improve accuracy, programming can be done by including the transmission losses.

\section{References}

[1]. Aruldoss Albert victorie, T. and Ebenezer Jeyakumar, A. (2004) "Hybrid PSP-SQP for economic dispatch with valve point effect", Electric power system research, vol.no.71, pp. 51-59.

[2]. Attaviriyanupap P., Hasegawa J., Kita H., and Tanaka E. (2002)"A hybrid EP and SQP for dynamic economic dispatch with nonsmooth incremental fuel cost function", IEEE Transactions on Power System, vol. 17, no. 2, pp. 411-416.

[3]. Aug and Zwe- Lee Gaing (2003) "Particles swarm optimization to solving the Economic Dispatch Considering the Generator constraints", IEEE Transactions on power systems, vol. 18, No. 3, pp.1157-1195.

[4]. Bentley, P. and Blackwell, T. "Don't push me! Collision-avoiding swarms," in Proc. IEEE Congr. Evol. Comput., May 2002, vol. 2, pp. 1691-1696.

[5]. Bhagwan Das, D. and Patvardhan, C. (1999) "Solution of Economic Load Dispatch using real coded Hybrid Stochastic Search", Electrc. Power Energy System, vol.no 21, pp. 165-170.

[6]. Boeringer, D.W. and Werner, D.H. "Particle Swarm Optimization Versus Genetic Algorithms for Phased Array Synthesis," IEEE Transactions on antennas and propagation, vol. 52, n. 3, pp. 771-779, March 2004. 
[7]. Boggs, P.T. and Tolle, J.W. (1995) "Sequential Quadratic Programming", Acta Numerica, Cambridge University Press, Cambridge, vol.no 4, pp. 1-52.

[8]. Chang C.S., Liew A.C., Srinivasan D., and Wen F. (1996) "A survey of evolutionary computing in power systems", IEEE Proceedings, pp. 35-41.

[9]. Chia-Feng Juang, “A Hybrid of Genetic Algorithm and Particle Swarm Optimization for Recurrent Network Design," IEEE Transactions On Systems, Man And Cybernetics-Part B: Cybernetics, vol. 34, n. 2, pp. 997-1006, April 2004.

[10]. Chowdhury, B.H. and Rahman, S. (2001) "A review of recent advances in economic dispatch", IEEE Transaction on Power System, vol.5, no.4, pp.1248-1259.

[11]. Fonseca N. and Miranda V. "New evolutionary particle swarm algorithm (EPSO) applied to voltage/VAR control," in Proc. 14th Power Syst. Comput. Conf., Jun. 2002.

[12]. Fukuyama Y., Genji T., Naka S., and Yura T., "A hybrid particle swarm optimization for distribution state estimation," IEEE Trans. Power Syst., pp. 60-68, Feb. 2003.

[13]. El-Dib A., El-Metwally M., Osman Z., and Youssef H., "Load flow solution using hybrid particle swarm optimization," in Proc. Int. Conf. Elect., Electron., Comput. Eng., Sep. 2004, pp. 742-746. 